\title{
Aversive cues fail to activate fos expression in the asymmetric olfactory-habenula pathway of zebrafish
}

\author{
Tagide N. deCarvalho ${ }^{1 *}$, Courtney M. Akitake ${ }^{1}$, Christine Thisse $^{2}$, Bernard Thisse $^{2}$ and \\ Marnie E. Halpern ${ }^{1 *}$
}

1 Department of Embryology, Carnegie Institution for Science, Baltimore, MD, USA

${ }^{2}$ Department of Cell Biology, Health Science Center, University of Virginia, Charlottesville, VA, USA

\section{Edited by:}

German Sumbre, Ecole Normale

Superieure, France

Reviewed by:

Minmin Luo, National Institute of

Biological Sciences, China

Suresh Jesuthasan, Duke/NUS

Graduate Medical School, Singapore

*Correspondence:

Tagide N. deCarvalho and Marnie E. Halpern, Department of

Embryology, Carnegie Institution for Science, 3520 San Martin Drive,

Baltimore, MD 21218, USA.

e-mail: decarvalho@ciwemb.edu;

halpern@ciwemb.edu
The dorsal habenular nuclei of the zebrafish epithalamus have become a valuable model for studying the development of left-right (L-R) asymmetry and its function in the vertebrate brain. The bilaterally paired dorsal habenulae exhibit striking differences in size, neuroanatomical organization, and molecular properties. They also display differences in their efferent connections with the interpeduncular nucleus (IPN) and in their afferent input, with a subset of mitral cells distributed on both sides of the olfactory bulb innervating only the right habenula. Previous studies have implicated the dorsal habenulae in modulating fear/anxiety responses in juvenile and adult zebrafish. It has been suggested that the asymmetric olfactory-habenula pathway (OB-Ha), revealed by selective labeling from an Ihx2a:YFP transgene, mediates fear behaviors elicited by alarm pheromone. Here we show that expression of the fam84b gene demarcates a unique region of the right habenula that is the site of innervation by Ihx2a:YFP-labeled olfactory axons. Upon ablation of the parapineal, which normally promotes left habenular identity; the fam84b domain is present in both dorsal habenulae and Ihx2a:YFP-labeled olfactory bulb neurons form synapses on the left and the right side. To explore the relevance of the asymmetric olfactory projection and how it might influence habenular function, we tested activation of this pathway using odorants known to evoke behaviors. We find that alarm substance or other aversive odors, and attractive cues, activate fos expression in subsets of cells in the olfactory bulb but not in the Ihx2a:YFP expressing population. Moreover, neither alarm pheromone nor chondroitin sulfate elicited fos activation in the dorsal habenulae. The results indicate that L-R asymmetry of the epithalamus sets the directionality of olfactory innervation, however, the Ihx2a:YFP OB-Ha pathway does not appear to mediate fear responses to aversive odorants.

Keywords: behavior, asymmetry, alarm pheromone, fos, fam84b

\section{INTRODUCTION}

The epithalamus of the teleost brain shows a high degree of leftright (L-R) asymmetry (Concha and Wilson, 2001; Kuan et al., 2007a; Signore et al., 2009); however, the functional significance of this specialization is unknown. In zebrafish, the pineal stalk emerges just to the left of the midline and the accessory parapineal is located to its left (Concha et al., 2000; Liang et al., 2000; Gamse et al., 2002). The adjacent paired habenular nuclei are comprised of dorsal and ventral nuclei, which correspond to the mammalian medial and lateral nuclei, respectively (Amo et al., 2010). The dorsal nuclei can be further divided into asymmetric subnuclei based on their distinct molecular properties (Gamse et al., 2003; Aizawa et al., 2005; Gamse et al., 2005) and the region of the interpeduncular nucleus (IPN) that they innervate (Agetsuma et al., 2010). Thus, the dorsal habenulae display striking differences in size, neuroanatomy, molecular properties, and connectivity.

The asymmetric properties of the zebrafish larval epithalamus arise in a step-wise manner, with Nodal signaling setting the directionality of the pineal complex (Concha et al., 2000; Liang et al., 2000) and the parapineal, which emerges on the left side, providing cues that differentiate the left dorsal habenula from the right (Concha et al., 2003; Gamse et al., 2003; Snelson et al., 2008; Regan et al., 2009). The dorsal habenular nuclei are part of an evolutionarily conserved conduction system between the limbic forebrain and the midbrain via efferent connections to the IPN (Herrick, 1948; Sutherland, 1982; Hikosaka et al., 2008; Bianco and Wilson, 2009). However, in zebrafish, habenular connections to the IPN do not show mirror image symmetry as in mammals (Ramón y Cajal, 1995). Instead, efferents from the left habenula project to the dorsal, intermediate, and ventral regions of the IPN, and the right habenula innervates only the ventral region (Gamse et al., 2005). Further supporting dorsoventral differences at the target, neurons in the dorsal IPN project to the griseum centrale, a region thought to be equivalent to the mammalian periaqueductal gray, whereas those in the ventral IPN innervate the median raphe nucleus (Agetsuma et al., 2010). L-R differences in habenular connectivity thus result in distinct limbic to midbrain pathways on each side of the brain. 
In mammals, the habenular region has been implicated in a wide range of behaviors including fear, sex, and feeding (Sutherland, 1982; Bianco and Wilson, 2009; Hikosaka, 2010; Okamoto et al., 2012). The role of the lateral/ventral habenular nuclei in the modulation of emotional behaviors has been intensively studied in rhesus monkey and humans [refer to Hikosaka (2010)]. The function of the medial/dorsal habenula is less well-understood; however, there is evidence that aversive stimuli or stress induce behavioral, biochemical and immunological responses (Silver et al., 1996; Carboni et al., 1998; Cirulli et al., 1998; Sugama et al., 2002; Wilhelm, 2011; Kobayashi et al., 2013).

Recent studies on zebrafish demonstrate that the dorsal habenulae regulate fear-related behaviors. Juveniles with the dorsal habenulae (and other structures) genetically ablated did not learn to avoid an aversive stimulus when paired with a conditional one (Lee et al., 2010). Inactivation of an asymmetric dorsal habenular subnucleus resulted in increased freezing behavior in adult zebrafish in response to a conditioned aversive stimulus (Agetsuma et al., 2010). These studies suggest that the dorsal habenula is an experience-dependent modulator of anxiety and fear-related decision-making (Okamoto et al., 2012).

A known stimulus that elicits fear in fish is "Schreckstoff" or fear substance, an array of chemicals exuded from injured skin, which functions as a predation signal for nearby individuals (Pfeiffer, 1977). Perception of such alarm pheromones in zebrafish provokes rapid darting or freezing, alternative behaviors in response to fear (Waldman, 1982; Speedie and Gerlai, 2008). Because of the dorsal habenula's involvement in modulating fear responses, it has been proposed that alarm pheromone may be the odorant cue for the asymmetrically projecting olfactory-habenula (OB-Ha) pathway (Concha et al., 2012). Furthermore, whole skin extract or glycosaminoglycan chondroitin, results in increased calcium signaling in the medio-dorsal portion of the posterior olfactory bulb (Mathuru et al., 2012), where lhx2a:YFP labeled mitral cells are located.

In the present study, we describe a unique domain in the right dorsal habenula characterized by expression of the fam $84 \mathrm{~b}$ gene and associated with a discrete neuropil density that corresponds to the site of innervating $\ln \times 2 a$ :YFP positive olfactory mitral cells. Expression of fam $84 b$ and the synaptic terminals of the lhx2a:YFP neurons are always found in the dorsal habenula that is contralateral to the parapineal. Following removal of the parapineal, fam $84 b$ expression appears in the left and the right dorsal habenulae and both receive innervation from the olfactory neurons, indicating that directional asymmetry of the epithalamus is sufficient to influence pre-synaptic input. To examine the function of the asymmetric OB-Ha pathway, induction of fos expression was used as a measure of neuronal activation in response to a variety of odorants. We find that adult zebrafish exposed to alarm substance extracted from skin, or to the purified component chondroitin sulfate, show robust fos activation in the olfactory bulb, but not in the lhx2a:YFP subpopulation or in the dorsal habenulae. The results show that the epithalamus directs the formation of an asymmetric telencephalic connection; however, substances that provoke fearful behaviors do not appear to activate this unique OB-HA pathway.

\section{MATERIALS AND METHODS ZEBRAFISH}

Zebrafish were housed at $27^{\circ} \mathrm{C}$ on a $14: 10 \mathrm{~h}$ light:dark cycle. Zebrafish used in this study were the wild-type $\mathrm{AB}$ strain (Walker, $1999)$ and the transgenic lines $T g(-10 l h \times 2 a: G A P-E Y F P)^{\mathrm{zf} 177}[$ formerly called $\operatorname{Tg}(\operatorname{lh} \times 2 a: g a p-Y F P)]$ and $\operatorname{Tg}(-10 \operatorname{lh} \times 2 a: S Y P-G F P)^{\mathrm{zf} 186}$ [formerly called $\operatorname{Tg}(\operatorname{lh} x 2 a: S y p-G F P)$; Miyasaka et al., 2009] and

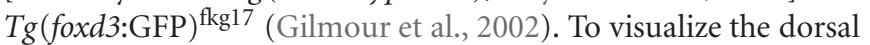
habenular nuclei, a transgenic reporter, $\mathrm{TgBAC}(\mathrm{gng} 8: \mathrm{Eco}$. NfsB2A-CAAX-GFP) (DKEY-18313 BAC; Genbank accession number CR450711) that is habenula-specific at $4-5$ days post-fertilization (dpf) (Akitake and Halpern, unpublished observations) was also employed. Maintenance of zebrafish and experimental procedures were carried out in accordance with the protocol approved by Institutional Animal Care and Use Committee. Naming of transgenic lines and zebrafish genes (see below) follow the nomenclature guidelines provided by the Zebrafish Model Organism Database (ZFIN).

\section{RNA in situ HYBRIDIZATION AND IMMUNOFLUORESCENCE}

The family with sequence similarity 84 , member $B$ (fam84b) gene was identified in an in situ hybridization screen of zebrafish cDNAs for their tissue-specific patterns of expression (Thisse and Thisse, 2004). The fam84b clone came from a cDNA library prepared from adult zebrafish kidney. Digoxigenin-(DIG) and fluorescein (FITC)-labeled RNA probes were synthesized using reagents from Roche Molecular Biochemical. The fam $84 b$ plasmid was linearized with NotI and transcribed with SP6 RNA polymerase. A clone of the $v$-fos FBJ murine osteosarcoma viral oncogene homolog gene (fos, formerly referred to as $c$-fos) was generated by amplifying a 602 base pair fragment from cDNA prepared from RNA of $3 \mathrm{dpf}$ larvae using forward and reverse primers (TCTCCTCTGTGGCGCCCTCC and GTCTGGAACCGAGCGAGCCG) and subcloning into the pCRII-TOPO vector (Invitrogen). For probe synthesis, the fos plasmid was linearized with BamHI and transcribed with T7 RNA polymerase. The orthodenticle homolog 5 (otx5) and potassium channel tetramerization domain containing 12.1 ( $k c t d 12.1$, formerly called leftover) probes were prepared as described (Gamse et al., 2002, 2003). A probe for the early growth response 1 (egrl) gene was synthesized as in Close et al. (2002).

Single and double label colorimetric RNA in situ hybridization experiments were performed as previously described for larvae (Gamse et al., 2002) and adult brain tissue (Gorelick et al., 2008). To enhance signal intensity, 5\% dextran sulfate (Millipore) was added to the hybridization buffer as in Lauter et al. (2011). Following the colorimetric reaction, adult brains were embedded in $4 \%$ low melting point agarose (SeaPlaque, Lonza) and $50 \mu \mathrm{m}$ coronal sections were collected using a vibratome (Leica VT1000S).

For fluorescent in situ hybridization alone or coupled with immunolabeling for yellow fluorescent protein (YFP)/green fluorescent protein (GFP) YFP/GFP, we followed the protocol described in Gamse et al. (2002) until the anti-DIG antibodyblocking step. Samples were placed in maleic acid buffer (MAB) with $2 \%$ blocking reagent (Roche) for $1 \mathrm{~h}$ according to Lauter et al. (2011). Anti-DIG-antibody conjugated with horseradish 
peroxidase (Roche) alone or together with anti-GFP rabbit antibody (Torrey Pines TP401) was diluted 1:500 in blocking solution and incubated at $4{ }^{\circ} \mathrm{C}$ overnight. Probes were visualized by tyramide signal amplification (TSA) reagents that were prepared as described (Vize et al., 2009). Anti-GFP antibody crossreacts with YFP. Reaction buffer contained 4-iodophenol (SigmaAldrich) and 2\% dextran sulfate. Tyramide-Cy5 was used for the first in situ hybridization probe. For double in situ hybridization, reacted samples were incubated in blocking solution for $1 \mathrm{~h}$ and then at $4^{\circ} \mathrm{C}$ overnight in diluted (1:500) anti-FITC-antibody conjugated with horseradish peroxidase (Roche). Tyramide-Cy3 was used for the second in situ hybridization probe. Adult brains were embedded in $4 \%$ low melting point agarose and $50 \mu \mathrm{m}$ coronal vibratome sections were collected prior to the TSA detection step. The sections were mounted in Prolong Gold or Slowfade Gold (Invitrogen) antifade mounting media. For immunolabeling following fluorescent in situ hybridization, reacted samples were placed in blocking solution (0.1\% Triton PBS with $10 \%$ sheep serum) for $1 \mathrm{~h}$ and then in diluted (1:500) goat anti-rabbit-Cy3 antibody (Jackson ImmunoResearch) at $4^{\circ} \mathrm{C}$ overnight.

\section{ODOR ASSAYS}

Alarm substance (hereinafter referred to as skin extract) was freshly prepared on the day of testing. Adult fish were immersed in an ice water slurry and decapitated. To prepare skin extract solution, the body was placed in $10 \mathrm{~mL}$ distilled water, the skin was abraded with 250 grit sandpaper and 15 shallow cuts were made using a protocol modified from Speedie and Gerlai (2008). Extracts were collected and pooled from 5 male and 5 female individuals $(100 \mathrm{~mL}$ total $)$ and filtered under vacuum using a $0.2 \mu \mathrm{m}$ filter (Nalgene).

Adult zebrafish (3-6 months) of both sexes were placed in $1 \mathrm{~L}$ breeding tanks (Aquatic Habitats) containing system water and acclimated for $1 \mathrm{~h}$ prior to odorant exposure. All experiments were performed with adults because larval zebrafish do not respond to alarm pheromone (Waldman, 1982). For alarm pheromone assays, single fish were placed in tanks separated by black paper. Solutions were delivered via a $5 / 32 \times 9 / 32 \mathrm{~mm}$ diameter tygon tube (Pennplex) entering below the water line. A $10 \mathrm{~mL}$ syringe filled with $2.5 \mathrm{~mL}$ of the skin extract or chondroitin sulfate solution was connected to the other end of the plastic tubing and the contents slowly injected into each tank for a final concentration of $0.2 \%$ skin extract or $1 \mu \mathrm{g} / \mathrm{ml}$ shark chondroitin sulfate (Sigma-Aldrich). Other ecologically and behaviorally relevant odors were tested in a similar manner; however, groups of 5 fish were placed in one tank for each odorant. Test chemicals were purchased through Sigma-Aldrich and used at a final concentration of $400 \mu \mathrm{M}$ for L-cysteine and putrescine and $40 \mu \mathrm{M}$ for cadaverine and trimethylamine. Ground fish flakes $(100 \mathrm{mg} / \mathrm{L}$; Tetramin Tropical Flakes), 0.1\% bile obtained from freshly killed tilapia (Orechromis mossambicus) and $0.25 \%$ of a conspecific extract, which was obtained by grinding and straining 10 freshly sacrificed adult zebrafish of both sexes into a $10 \mathrm{ml}$ of distilled water were also tested. Control individuals received only distilled water. Following odorant exposure, fish were held in the experimental tanks for $30 \mathrm{~min}$, then immersed in an ice water slurry and decapitated. Brains were immediately dissected in cold PBS and fixed in $4 \%$ paraformaldehyde at $4^{\circ} \mathrm{C}$ overnight.
As a positive control, the glutamate receptor agonist, kainic acid, was used to elicit fos expression in $l h x 2 a$ :YFP labeled mitral cells. Primary sensory neurons excite mitral cells through AMPA and kainic ionotropic glutamate receptors (Tabor and Friedrich, 2008); therefore, treatment with kainic acid is expected to induce neural activity in the olfactory bulb. Adult fish were anesthetized with 4\% tricaine (ethyl 3-aminobenzoate methanesulfonate solution; Sigma-Aldrich; Westerfield, 2000) and placed on a damp sponge. Kainic acid (Tocris Bioscience) was injected into the peritoneal cavity via a syringe (28G, Beckton Dickinson) at $10 \mathrm{mg}$ per $\mathrm{kg}$ body mass in phosphate buffered saline (PBS). Control fish were injected with PBS only. The injected fish were placed in a recovery tank for $30 \mathrm{~min}$ and treated as above.

\section{PARAPINEAL ABLATION}

Parapineal ablation was performed on $T g(f o x d 3: G F P)^{\mathrm{fkg} 17}$, $T g(f o x d 3: G F P)^{\mathrm{fkg} 17} ; \operatorname{Tg}(-10 l h \times 2 a: G A P-E Y F P)^{\mathrm{zf177}}$ or $T g(f o x d 3$ : GFP $)^{\mathrm{fkg} 17}$; $\mathrm{Tg}(-10 \operatorname{lh} \times 2 a: S Y P-G F P)^{\mathrm{zf} 186}$ embryos at 2 or $3 \mathrm{dpf}$. Embryos were anesthetized with $0.04 \%$ tricaine (ethyl 3 -aminobenzoate methanesulfonate solution; Sigma-Aldrich; Westerfield, 2000) and positioned dorsal side up in $1.2 \%$ low melting-temperature agarose under a $40 \times$ water immersion lens. A scanning beam ( $885 \mathrm{~nm}$ excitation) from a multiphoton Ti:Sapphire laser (MaiTai HP, Spectra-Physics) mounted on a Leica SP5 confocal microscope was focused on the parapineal. Cell ablation was achieved by scanning with a $30-40 \%$ filter wheel (power) setting for 1 to 3 two-second passes. The extent of ablation was monitored with a $10 \%$ filter wheel setting after each scan pass until GFP was no longer detected. Successful ablation of the parapineal was re-evaluated by fluorescence microscopy prior to live imaging or fixation. In control larvae, cells in the brain were ablated on the opposite side of the pineal or adjacent to the parapineal.

\section{IMAGING}

Bright-field images were collected using an Axiocam HRc digital camera mounted on an Axioskop (Carl Zeiss). Fluorescent images were captured using a Zeiss Imager.Z1 microscope equipped with an AxioCam MRm digital camera or with a Leica SP5 confocal microscope. Images were pseudocolored and three-dimensional projections were generated using Imaris (Bitplane) or Axiovision (Carl Zeiss) software.

\section{RESULTS}

\section{Ihx2a: YFP OLFACTORY NEURONS PROJECT TO A fam84b-EXPRESSING SUBREGION OF THE RIGHT DORSAL HABENULA}

The dorsal habenular nuclei exhibit L-R differences in gene expression and in the extent of dense neuropil (Concha et al., 2000; Gamse et al., 2003, 2005), while the ventral nuclei are bilaterally symmetrical (Amo et al., 2010). The fam84b gene, which encodes a breast cancer membrane-associated protein (Adam et al., 2003) is transcribed bilaterally in the ventral habenulae, but is also expressed in an unusual pattern in only the right dorsal habenula (Figures 1A and 2A). The fam $84 b$ domain is within the lateral subnucleus of the right dorsal habenula (Figure 1B), defined by expression of the kctd12.1 gene (Aizawa et al., 2005; Gamse et al., 2005). The medio-lateral designation of habenular subnuclei is based on their position in the adult brain, which 

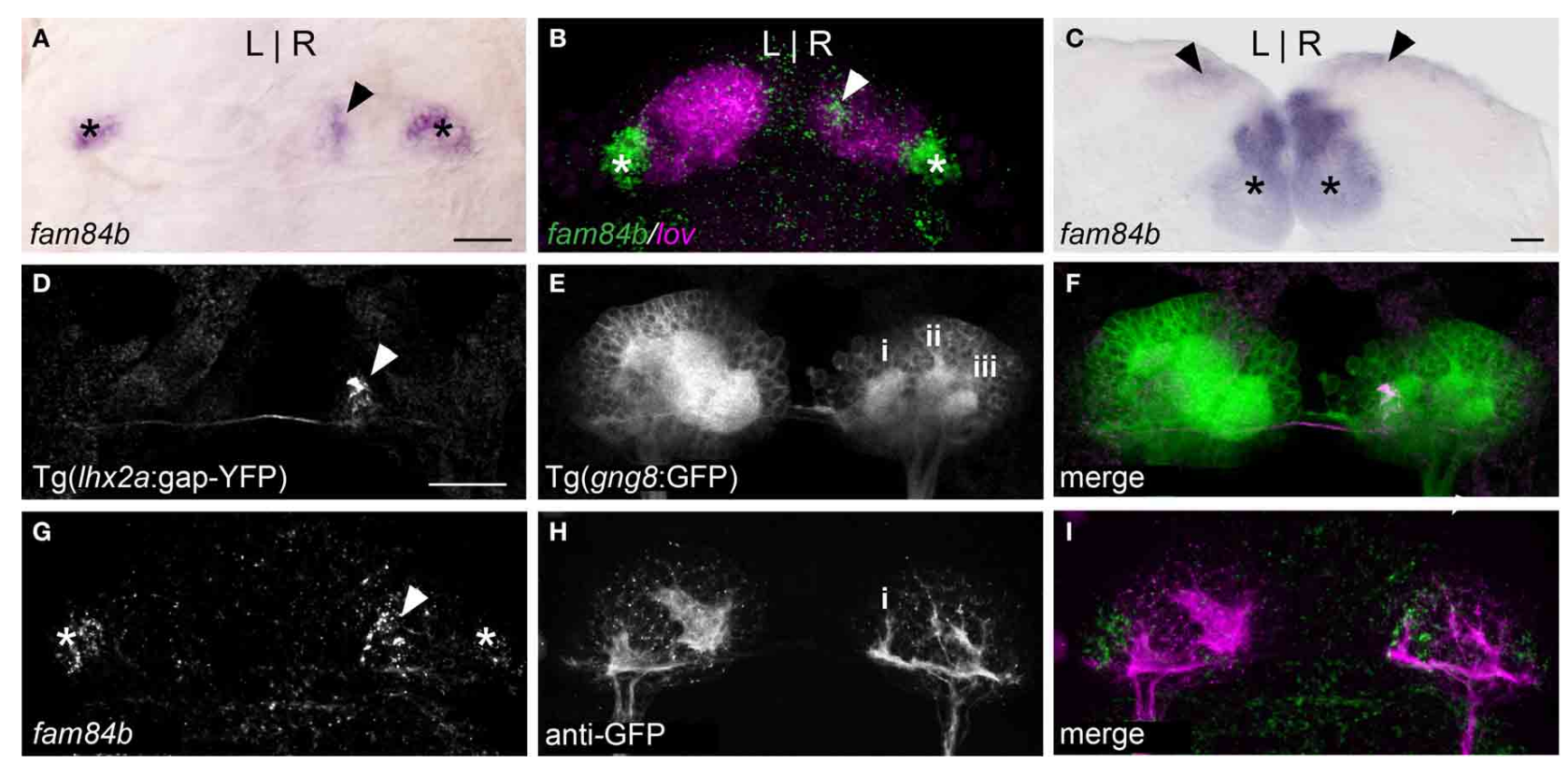

FIGURE 1 | A unique subnucleus in the right dorsal habenula of larval zebrafish. (A) The fam84b gene is expressed in a medial domain in the right dorsal habenula (black arrowhead) and bilaterally in the ventral habenulae (asterisks in $\mathbf{A - C , G ) . ~ ( B ) ~ T h e ~ r i g h t - o n l y ~ f a m 8 4 b ~ d o m a i n ~ ( w h i t e ~ a r r o w h e a d ) ~ i s ~}$ located within the kctd 12.1 (lov)-expressing subnucleus, as demonstrated by fluorescent in situ hybridization. (C) fam $84 \mathrm{~b}$ is expressed bilaterally in the adult dorsal habenulae (black arrowheads). (D-F) Live imaging of
Tg(gng8: $n f S B-C A A X-G F P)^{c 375}$ larva reveals that axons of Ihx2a:YFP labeled olfactory mitral cells (white arrowhead in D) terminate at the medial neuropil (i in $\mathbf{E})$ of the right habenula. More lateral neuropil clusters are indicated (ii,iii). (G-I) The medial neuropil cluster (i) visualized by anti-GFP immunofluorescence in Tg(gng8:nfsB-CAAX-GFP) ${ }^{\mathrm{c} 375}$ larva is closely associated with the fam84b expression domain (white arrowhead in $\mathbf{G}$ ) revealed by fluorescent in situ hybridization. All larvae $4 \mathrm{dpf}$; scale bars $=50 \mu \mathrm{m}$. differs from the larva due to extensive reorganization that occurs during development (Amo et al., 2009). Expression of fam84b is bilateral in the adult, but remains more extensive in the right dorsal habenula (Figure 1C).

Accumulations of neuropil differ between the left and right dorsal habenulae of larval zebrafish, as visualized by immunolabeling with antibodies against acetylated $\alpha$-Tubulin (Concha et al., 2000; Taylor et al., 2011) or Synaptic Vesicle Protein 2 (SV2) (Hendricks and Jesuthasan, 2007; Miyasaka et al., 2009), and by labeling of membrane-tagged GFP in live $T g(g n g 8: n f s B$ $C A A X-G F P)^{\mathrm{c} 375}$ larvae. The left side has an expanded neuropil that extends the width of the dorsal habenula, while the right dorsal habenula has three more distinct, small clusters (Figure 1E). The most medial cluster corresponds to the site of innervation of lhx2a:YFP labeled axons from the olfactory bulb (Figures 1D-F) and overlaps with the fam $84 b$-expressing cells (Figures 1G-I). The convergence of asymmetric pre-synaptic olfactory input, a discrete neuropil cluster and restricted fam $84 b$ expression suggests that the right dorsal habenula contains a functionally unique subnucleus.

\section{DIRECTIONALITY OF OLFACTORY INNERVATION IS DETERMINED BY L-R ASYMMETRY OF THE EPITHALAMUS}

Parapineal cells originate from the pineal anlage at $28 \mathrm{~h}$ postfertilization (hpf) and migrate to the left side of the brain in $>95 \%$ of embryos (Concha et al., 2003; Gamse et al., 2003). By $40 \mathrm{hpf}, k c t d 12.1$ is strongly expressed in the presumptive left habenula and to a far lesser extent in the right. However, when the parapineal is found on the right side of the brain, either spontaneously $(<5 \%)$ or following perturbation of Nodal-related signaling (Facchin et al., 2009), the asymmetric kctd12.1 expression pattern is L-R reversed. Loss of the parapineal, either by laser-mediated cell ablation (Concha et al., 2003; Gamse et al., 2003) or the consequence of mutation (Snelson et al., 2008; Regan et al., 2009) results in symmetric development of the habenulae, with both the left and right nuclei exhibiting properties of the right habenula. Accordingly, when the parapineal is situated on the right side of the brain, fam $84 b$ is expressed in the left dorsal habenula ( $n=3$, Figure 2B) and, following ablation of the parapineal, fam $84 b$ domains are found bilaterally in both dorsal habenulae (control $n=4$, Figures 3A,B; ablated $n=5$, Figures 3C,D).

We examined whether altered directional asymmetry of the epithalamus also influences the connectivity of $\operatorname{lh} x 2 a$ :YFP labeled mitral cells. L-R reversal of parapineal position correlated with the olfactory bulb mitral cells innervating the left habenula rather than the right (Figures 2C,D). Compared to control ablated larvae with unilateral input to the right habenula (Figures $3 \mathbf{E}-\mathbf{G}$ ), lhx2a:YFP efferents innervate both dorsal habenulae in larvae with an ablated parapineal $(n=14$, Figures $3 \mathbf{H}-\mathbf{J})$. These results indicate that L-R asymmetry of the epithalamic region directs olfactory connectivity. The bilateral projections likely form functional synapses, as evidenced by the localization of synaptic vesicles using $\operatorname{Tg}(-10 \operatorname{lh} \times 2 a: S Y P-G F P)^{\mathrm{zf} 186}$, in which a synaptophysin-YFP fusion protein is expressed under the control of the $\ln \times 2 a$ cis-regulatory elements (Miyasaka et al., 2009). 


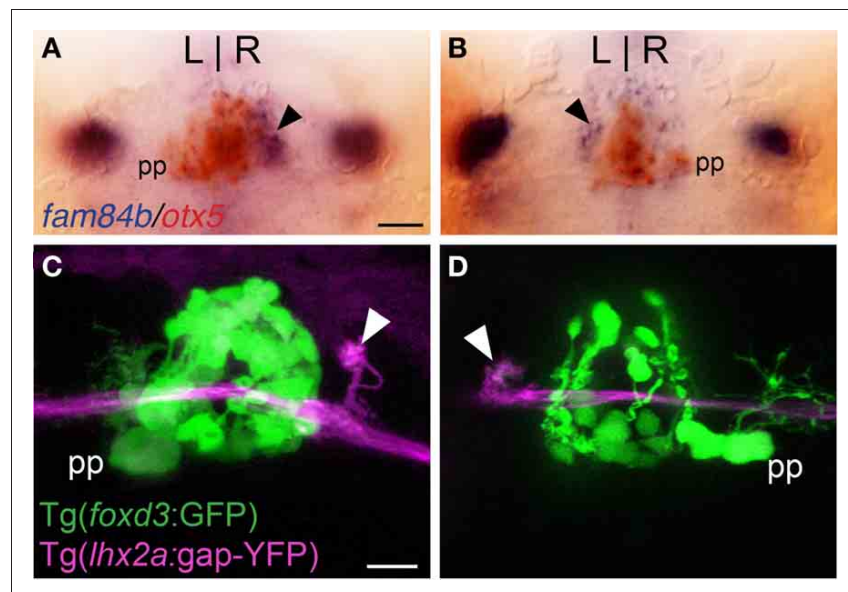

FIGURE 2 | Asymmetric olfactory input is contralateral to the parapineal. (A,B) Larvae with the parapineal $(\mathrm{pp})$ on the left of the pineal anlage exhibit fam84b expression (blue) in the right dorsal habenula (black arrow). Conversely, spontaneous L-R reversal of parapineal position corresponds with fam84b expression on the left. The pineal complex expresses the otx5 gene (red), revealed by double label in situ hybridization methods. (C,D) Live imaging of $T g(f o x d 3: G F P)^{\mathrm{fkg} 17}$; $T g$ (-10lhx2a:GAP-EYFP) zf177 larvae. When the parapineal is on the left, Ihx2a:YFP labeled terminals (white arrowhead) are found in the right dorsal habenula. With L-R reversal of epithalamic asymmetry, olfactory axons terminate in the left dorsal habenula. All larvae $4 \mathrm{dpf}$. Scale bars $=50 \mu \mathrm{m}$ for $(\mathbf{A}, \mathbf{B})$ and $25 \mu \mathrm{m}$ for $(\mathbf{C}, \mathbf{D})$.

Clusters of synaptic vesicles were found in axon terminals at both dorsal habenulae ( $n=3$, Figures $3 \mathbf{N}, \mathbf{O}$ ). Ablation of the parapineal at a later developmental stage $(77-80 \mathrm{hpf})$, following the establishment of the lhx2a:YFP olfactory-right habenula projection, also resulted in innervation of both dorsal habenulae $(n=$ 13, Figures $\mathbf{3 K}-\mathbf{M}$ ). This reveals that the parapineal is required for the maintenance of dorsal habenular L-R identity, and that the OB-Ha pathway is highly plastic.

\section{ALARM PHEROMONE DOES NOT STIMULATE THE OB-Ha PATHWAY}

The dorsal habenulae have been implicated in modulating fear responses. Therefore, we examined whether substances known to provoke anxious behaviors in fish activate the asymmetric OB-Ha pathway in adult zebrafish. We used induction of fos expression as an indirect read-out of neural activity. This approach has been successfully applied in mammals to map the habenular response to a variety of stimuli (e.g., Kazi et al., 2004; Zhang et al., 2005; Paul et al., 2011) and the response to odorants in the olfactory bulb (e.g., Guthrie et al., 1993; Sallaz and Jourdan, 1993; Guthrie and Gall, 1995). We also examined expression of egr1 but it was less robust than fos and was not detected in the olfactory bulb in response to chondroitin sulfate ( $n=9$, data not shown) or vehicle control $(n=5)$.

Exposure to skin extract $(n=11$, Figure 4A) or purified shark chondroitin sulfate ( $n=4$, Figure 4C) did not elicit higher fos expression in the right dorsal habenula above control levels $(n=5$, Figure 4B and $n=6$, Figure 4D). In both treatment groups, a few fos positive neurons were found in the right habenula, but comparable expression was also observed in controls, suggesting that baseline fos induction is due to stimulusunrelated activity caused by the experimental paradigm.

We also examined the response to chondroitin sulfate in the olfactory bulb. The olfactory bulb is comprised of several layers and, in zebrafish, mitral cell bodies reside within the glomerular and superficial inner cell layers while the interneuronal granule cells are found in a deeper position (Fuller et al., 2006). We observed that chondroitan sulfate treated individuals exhibited higher levels of fos expression in all regions $(n=4$, Figure $4 \mathbf{E})$ compared to controls ( $n=6$, Figure 4F). However, fos transcripts were never detected in lhx2a:YFP labeled mitral cells (chondroitin sulfate $n=8$, control $=3$, Figures $4 \mathbf{G}, \mathbf{H}$ ). In contrast, many olfactory bulb neurons including the $\operatorname{lh} \times 2 a$ :YFP mitral cell subpopulation were activated with the glutamate receptor agonist kainic acid (Figures 4I-L). The centrally located fos positive cells are granule cells, whereas those found in the same layer or directly adjacent to $\operatorname{lh} x 2 a$-expressing neurons are most likely mitral cells.

\section{ECOLOGICALLY RELEVANT ODORS STIMULATE NON-Ihx2a:YFP OLFACTORY BULB NEURONS}

All odorant treatments elicited a qualitatively higher fos response above negative control throughout the olfactory bulb region but not in $l$ hx2a:YFP labeled mitral cells (data not shown, putrescine $n=4$, cadaverine $n=3$, trimethylamine $n=3$, L-cysteine $n=4$, fish flakes $n=5$, conspecific extract $n=3$, Tilapia bile $n=4$ ).

\section{DISCUSSION}

The connection from primary sensory neurons in the olfactory epithelium to secondary mitral cells in the olfactory bulb that project directly to the right habenula provides a defined pathway to study the establishment and behavioral significance of L-R asymmetry in the vertebrate brain.

Previous studies demonstrated that the parapineal influences the identity of the adjacent dorsal habenula. When the parapineal is located to the right of the pineal, rather than at its typical left-sided position, the directionality of habenular L-R asymmetry is reversed. In the absence of the parapineal, the left and right dorsal habenular nuclei develop similarly, with gene expression patterns, neuroanatomical organization, and restricted efferent connections to the IPN characteristic of the right nucleus. Accordingly, we find that the parapineal also affects the formation of a subnucleus in the right habenula, defined by expression of $f a m 84 b$ and the absence of the olfactory cell adhesion molecule (OCAM; Miyasaka et al., 2009). This subnucleus is found within a region designated as the lateral subnucleus of the dorsal habenula (Aizawa et al., 2005). It also corresponds with the location of a coherent neuropil cluster and the site of axon terminals of lhx2a:YFP olfactory mitral cells. With a L-R reversal in parapineal position, this subnucleus is now found in the left dorsal habenula. Following ablation of the parapineal, fam $84 b$ expression is present bilaterally and both the left and right habenular nuclei are innervated by the lhx2a:YFP neuronal population. These findings indicate that L-R asymmetry of the epithalamic region is sufficient to direct telencephalic input. Thus, a small difference in one region of the brain (e.g., the parapineal) not only influences the development of neighboring structures (e.g., the 

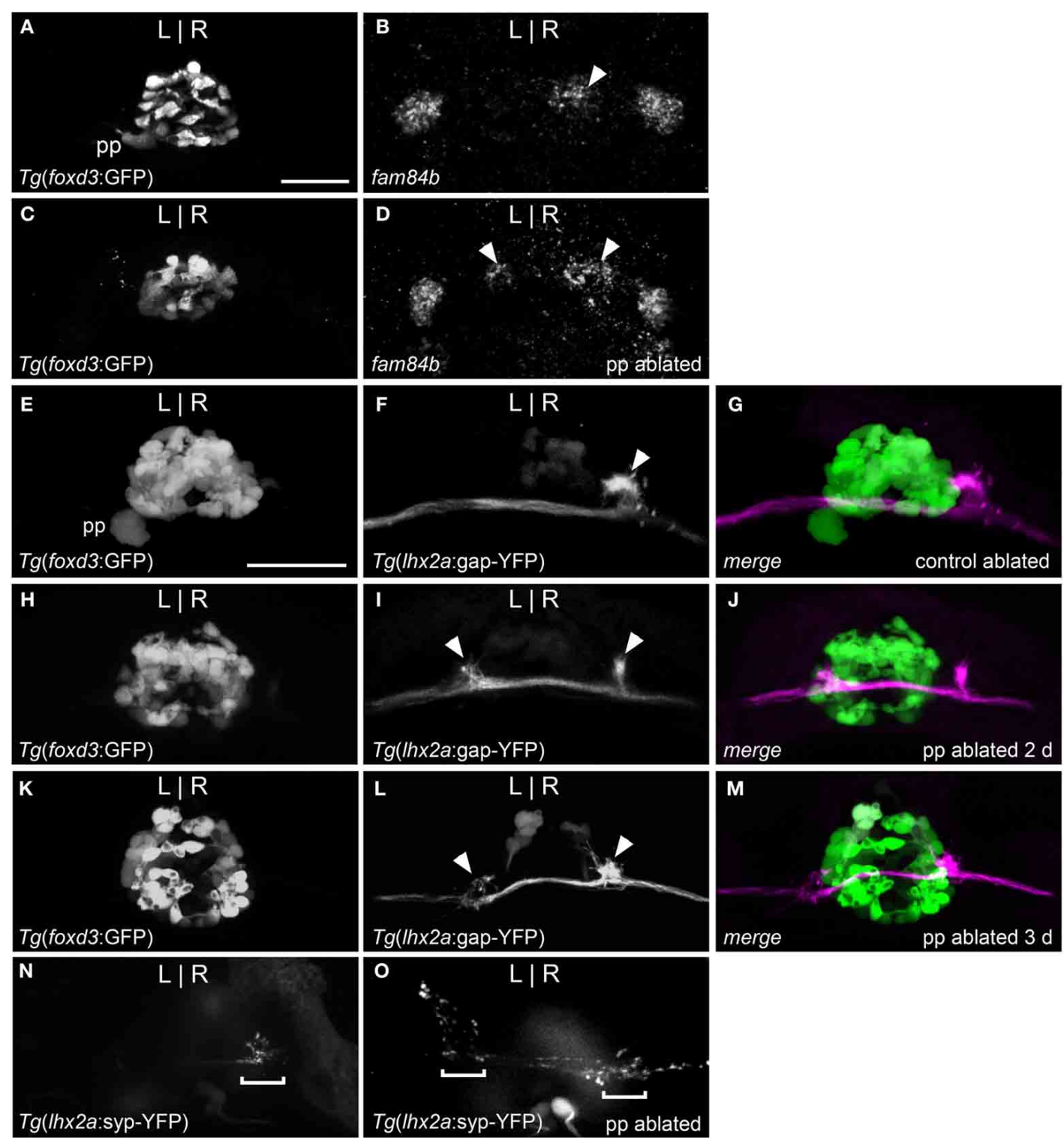

FIGURE 3 | Parapineal ablation results in symmetric OB-Ha pathway. (A) Live imaging of $T g(f o x d 3: G F P)^{f k g} 17$ larva, with parapineal (pp) situated to the left of the pineal. (B) Fluorescent in situ hybridization reveals fam84b transcripts in the right dorsal habenula (white arrow) and bilaterally in the ventral habenulae at $4 \mathrm{dpf}$. (C) Parapineal ablation at $2 \mathrm{dpf}$ results in (D) bilateral fam84b domains (white arrowheads) in the dorsal habenulae. (E-G) Ihx2a:YFP mitral cells (white arrowhead) innervate the right habenula of control-ablated larvae, but $\mathbf{( H )}$ in the absence of the parapineal, they terminate $\mathbf{( I , J )}$ at both habenulae (white arrowheads in $\mathbf{I})$. (K-M) Parapineal ablation at $3 \mathrm{dpf}$ also results in bilateral $/ h \times 2 a$ :YFP projections (white arrowheads in $\mathbf{L}$ ). (N) Region of synaptic vesicle density (demarcated by bracket) in the right dorsal habenula of $\mathrm{Tg}(-10 \mathrm{lh} \times 2 \mathrm{a}: S Y P-G F P)^{\text {zf186 }}$ larva is found $(\mathbf{O})$ bilaterally following parapineal ablation. All images, except for (B,D) are of live larvae at $4 \mathrm{dpf}, 5 \mathrm{dpf}(\mathbf{K}-\mathbf{M})$ and $6 \mathrm{dpf}(\mathbf{N}, \mathbf{O})$. Scale bars $=40 \mu \mathrm{m}$. dorsal habenulae), but also has farther-reaching consequences on pre-synaptic as well as post-synaptic neurons.

The molecular mechanisms that direct the asymmetric connection between lhx2a:YFP mitral cells and the right habenula have yet to be determined. One possibility is unilateral expression of an axon guidance molecule. The neuropilin receptor $1 a$ (nrpla) gene, for example, is expressed only by neurons in the left dorsal habenula (Kuan et al., 2007b). Its ligand, Sema3D, is produced in the midline rostral to the IPN and is important for guiding left habenula neurons to the dorsal region of the
IPN (Kuan et al., 2007b). Innervation of the right dorsal habenula by $l h x 2 a$ :YFP mitral cells is unaltered following depletion of Nrpla (deCarvalho and Halpern, unpublished observations), suggesting that formation of the asymmetric OB-Ha pathway does not rely on repulsive Sema3D-Nrp1 signaling. An alternative hypothesis is that the fam $84 b$-expressing cells provide an attractive signal to guide lhx2a:YFP axons to or stabilize their synapses at the right habenula. Fam84b (also known as breast cancer membrane protein 101 and NSE2) encodes a protein of unknown function that interacts with $\alpha 1$-Catenin and is localized to the 

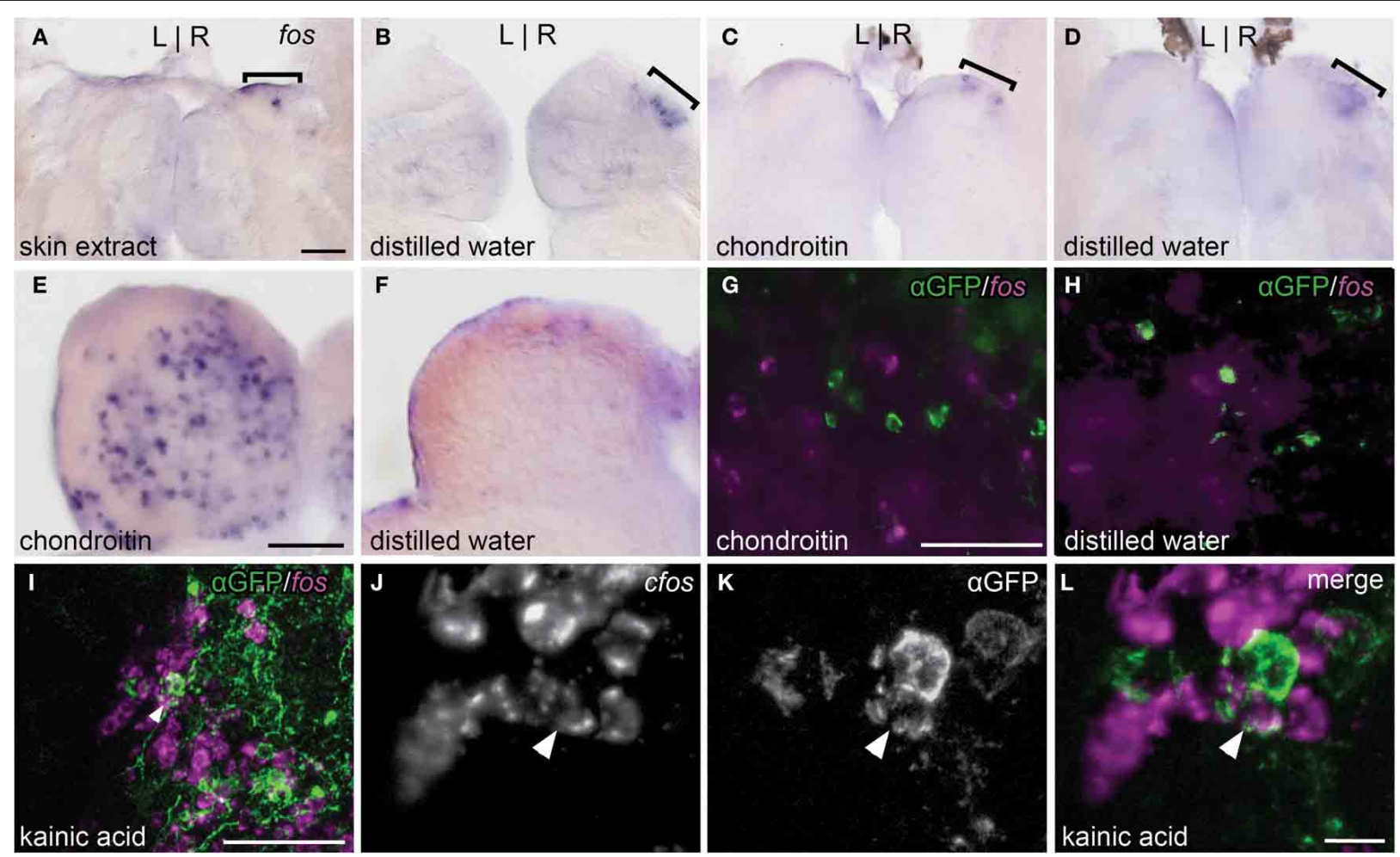

FIGURE 4 | Alarm substances do not stimulate the asymmetric OB-Ha pathway. (A,B) Skin extract does not elicit higher fos expression (brackets) than controls in the dorsal habenula. (C,D) Chondroitin sulfate also does not activate fos expression above controls in the dorsal

habenula. (E,F) Robust induction of fos expression in the olfactory bulb is observed following chondroitin sulfate exposure. (G,H) fos transcripts

revealed by fluorescent in situ hybridization do not colocalize with immunolabeled Ihx2a:YFP neurons in the olfactory bulb. (I) Kainic acid induces fos expression in a subset of Ihx2a:YFP labeled olfactory neurons. (J-L) Higher magnification of (I), with double labeled neuron indicated (white arrowhead). Coronal sections $(50 \mu \mathrm{m})$ of adult brains. Scale bars $=40 \mu \mathrm{m}$ for $(\mathbf{A}-\mathbf{D}),(\mathbf{E}, \mathbf{F}),(\mathbf{G}, \mathbf{H})$, and $\mathbf{( J - L )}$, and $10 \mu \mathrm{m}$ for (I)

membrane in regions of cell-cell contact (Adam et al., 2003). However, Fam84b itself is unlikely to be the guidance cue for innervating $l h x 2 a$ :YFP axons as the appearance of fam $84 b$ transcripts follows rather than precedes olfactory axon outgrowth to the right habenula (deCarvalho and Halpern, unpublished observations). Although the cues that establish the asymmetric OB-Ha projection are unknown, localization of synaptic vesicles in axonal terminals at the right habenula strongly suggest that this is a functional connection. Confirmation of laterality in neuronal activity will come from transgenic approaches, such as selective optogenetic activation of the lhx2a-expressing subpopulation with expression of the calcium indicator GCaMP (e.g. Muto et al., 2011; Ahrens et al., 2012; Akerboom et al., 2012).

The identity of the $l h x 2 a$ :YFP-expressing olfactory neurons does not lend much insight as to the odorant cues that stimulate this pathway. The $l h x 2 a$ :YFP labeled primary sensory neurons are a subpopulation of crypt cells, one of three types of olfactory sensory neurons (OSNs) defined by their morphology in fish (Hansen and Zeiske, 1998). Crypt cells are relatively sparse compared to other OSNs (Hansen and Finger, 2000; Schmachtenberg, 2006) and, in zebrafish, appear to be nearly homogenous in their expression of the olfactory receptor gene, ora4 (Oka et al., 2012). Ligands for the Ora4 receptor are unknown, although there is evidence in the trout that crypt cells are most sensitive to sexual pheromones (Vielma et al., 2008; Bazaes and Schmachtenberg, 2012). The restricted expression of $\ln x 2 a$ coupled with the fact that $\sim 10 \%$ of OSNs are ora 4 negative, suggests that crypt cells are a diverse population and that the $\mathrm{Ob}-\mathrm{Ha}$ pathway may sense a distinct odorant cue.

Because the lhx2a:YFP olfactory projections are located in an region of the $\mathrm{OB}$ responsive to the alarm pheromone component, chondroitin sulfate (Mathuru et al., 2012), and the dorsal habenulae have been implicated in the modulation of fear (Agetsuma et al., 2010; Lee et al., 2010), it was proposed that the asymmetric OB-Ha pathway in zebrafish mediates avoidance behavior elicited by alarm substances (Concha et al., 2012). To test this hypothesis, we measured fos expression in the olfactory bulb and dorsal habenulae following exposure to a variety of odorant cues. Induction of immediate early gene expression, such as fos, has been widely used as a read-out of olfactory stimulation in rodents (e.g., Guthrie et al., 1993; Sallaz and Jourdan, 1993; Guthrie and Gall, 1995; Okuyama et al., 2011; Bepari et al., 2012). In zebrafish, fos expression was an effective measure of neural activity upon light avoidance; (Lau et al., 2011) and in pharmacologically induced seizures (Baraban et al., 2005).

We found that a variety of complex and purified odorants produced robust fos expression in the olfactory bulb of adult 
zebrafish, supporting the validity of the assay. However, contrary to their proposed role, neither alarm pheromone nor chondroitin sulfate alone elicited fos activation in the lhx2a:YFP OB-Ha pathway. We did not detect colocalization of fos transcripts in lhx2a:YFP labeled mitral cells nor did we observe fos activation in the right habenula above control levels. We tested other odors known to be aversive or attractive cues, including polyamines that have been shown to elicit olfactory responses in fish (Friedrich and Korsching, 1998; Michel et al., 2003; Rolen et al., 2003) and may serve as feeding cues (Rolen et al., 2003). Additional attractive odors tested were food, conspecifics and bile, which is a social cue (Doving et al., 1980; Polkinghorne et al., 2001). We also examined the response to L-cysteine because zebrafish show strong avoidance to this odor (Vitebsky et al., 2005) and relatively high sensitivity compared to other amino acids (Michel and Lubomudrov, 1995). In all cases, fos expression was observed in cells in the OB but not in the lhx2a:YFP subpopulation. Thus, the lhx2a:YFP labeled pathway appears to be unresponsive to ecologically relevant odor cues known to elicit behavior in fish. Consistent with these findings, we also did not detect fos expression in the right dorsal habenula above baseline levels. Curiously, fos expression was frequently observed in the right habenula of controls as well as individuals exposed to odorants, suggesting that this activity was caused by some aspect of the experimental set-up or handling of the adult fish.

The most likely explanation for the absence of fos activation in $\ln x 2 a-Y F P$ olfactory neurons is that a relevant odor was not tested. However, it is also possible that subcomponents of complex odorants such as fish flakes or conspecific extract masked or antagonized an activating cue (e.g., specific amino acids). Purified amino acids and specific components of sexual pheromones have been shown to elicit responses in the crypt cells of other fish species (Vielma et al., 2008; Bazaes and Schmachtenberg, 2012).

Alternatively, although the glutamate agonist kainic acid induced fos expression in lhx2a:YFP mitral cells, the fos response to odorants may be less robust in these neurons. Several functional mapping studies of the olfactory bulb in other species have demonstrated that mitral cells are competent to activate transcription of fos or other immediate early genes; however, expression is often reduced and less extensive than in the granule cell interneurons (e.g., Guthrie et al., 1993; Sallaz and Jourdan, 1993; Guthrie and Gall, 1995). Transcriptional activation is also known to differ in sensitivity among immediate early genes (Isogai et al., 2011; Bepari et al., 2012). For this reason, we examined expression of another immediate early gene, egr 1, but in contrast to fos, egrl transcripts were not detected in the olfactory bulb following exposure to chondroitin sulfate. An alternative method may be required to assay neuronal activity in the asymmetrically projecting mitral cells, such as the use of genetically-encoded $\mathrm{Ca}^{2+}$ indicators.

It is also possible that the $\operatorname{lh} x 2 a-Y F P$ labeled mitral cells consist of two distinct subpopulations, those that either express fos in response to kainic acid or do not. Neuroanatomical tracing of individual $l h \times 2 a-Y F P$ mitral cells indicates that a fraction may terminate in the telencephalon (Miyasaka et al., 2009), whereas the majority projects to the right habenula. Whether these alternative projection patterns correspond to neurons with different sensitivities to fos activation remains to be determined.

Lateralization of olfactory systems is prevalent in invertebrates and appears to function either as a means to enhance odor discrimination or facilitate olfactory learning. For example, in Caenorhabditis elegans, bilaterally-paired sensory neurons express different olfactory receptors on the left and right side of the head (Wes and Bargmann, 2001). Asymmetry at the molecular level allows the worm to sense multiple odors using a limited number of OSNs. In the honeybee, individuals trained with their right antenna perform better on olfactory memory tasks than those trained with the left antenna (Letzkus et al., 2006). The right antenna seems to mediate short-term olfactory memory while the left is specialized for long-term memory retention (Rogers and Vallortigara, 2008). The higher number of sensilla on the right antennae compared to the left may account for lateralized olfaction in honeybees (Frasnelli et al., 2010). A recent study in Drosophila demonstrates that L-R differences may be quite subtle, in that differential neurotransmitter release between the ipsilateral and contralateral projections of single OSNs enables directional sensing of localized odorants (Gaudry et al., 2013).

Although the significance of asymmetry in zebrafish olfactory projections remains to be demonstrated, it may facilitate differential processing of stimuli as in invertebrate models. Several teleost species are known to exhibit L-R differences in their olfactory system. In flatfish such as the winter flounder, the upward facing right olfactory pathway from the olfactory epithelium through the telencephalon is significantly larger in volume (Prasada Rao and Finger, 1984) and L-R glomerular organization of the olfactory bulb is highly asymmetric in the turbot (Doldan et al., 2011). Studies of olfactory sensitivity in the Senegalese sole demonstrate tuning between the left and right olfactory epithelia to several different odorants (Velez et al., 2005, 2007).

The discovery of a specialized subnucleus in the right dorsal habenula of the zebrafish larva that is the site of olfactory innervation could provide important clues as to the downstream circuitry that mediates a lateralized response to environmental cues. The finding that directional asymmetry of the developing epithalamus guides the formation of this subnucleus reveals an unsuspected plasticity in olfactory connectivity and suggests that local L-R differences may have widespread consequences on neural pathways throughout the brain.

\section{ACKNOWLEDGMENTS}

We thank Ana Quintanal and Lindsey Knapp for technical assistance, Yi-Lin Yan and John Postlethwait for providing the egrl plasmid, Philippe Mourrain for fos primers, and Erik Duboué for helpful comments on the manuscript. Tagide N. deCarvalho is a recipient of a Ruth L. Kirschstein National Research Service Award (F32MH091980). This study was supported by funds to Christine Thisse and Bernard Thisse from the University of Virginia and by a grant to Marnie E. Halpern from the National Institutes of Health (2R01HD042215). 


\section{REFERENCES}

Adam, P. J., Boyd, R., Tyson, K. L., Fletcher, G. C., Stamps, A., Hudson, L., et al. (2003). Comprehensive proteomic analysis of breast cancer cell membranes reveals unique proteins with potential roles in clinical cancer. J. Biol. Chem. 278, 6482-6489.

Agetsuma, M., Aizawa, H., Aoki, T., Nakayama, R., Takahoko, M., Goto, M., et al. (2010). The habenula is crucial for experience-dependent modification of fear responses in zebrafish. Nat. Neurosci. 13, 1354-1356.

Ahrens, M. B., Li, J. M., Orger, M. B., Robson, D. N., Schier, A. F., Engert, F., et al. (2012). Brain-wide neuronal dynamics during motor adaptation in zebrafish. Nature 485 , 471-477.

Aizawa, H., Bianco, I. H., Hamaoka, T., Miyashita, T., Uemura, O., Concha, M. L., et al. (2005). Laterotopic representation of left-right information onto the dorso-ventral axis of a zebrafish midbrain target nucleus. Curr. Biol. 15, 238-243.

Akerboom, J., Chen, T. W., Wardill, T. J., Tian, L., Marvin, J. S., Mutlu, S., et al. (2012). Optimization of a GCaMP calcium indicator for neural activity imaging. J. Neurosci. 32, 13819-13840.

Amo, R., Aizawa, H., Takahashi, R., Kobayashi, M., Takahoko, M., Aoki, T., et al. (2009). Identification of the zebrafish ventral habenula as a homologue of the mammalian lateral habenula. Neurosci. Res. 65, S227.

Amo, R., Aizawa, H., Takahoko, M., Kobayashi, M., Takahashi, R., Aoki, T., et al. (2010). Identification of the zebrafish ventral habenula as a homolog of the mammalian lateral habenula. J. Neurosci. 30, 1566-1574.

Baraban, S. C., Taylor, M. R., Castro, P. A., and Baier, H. (2005). Pentylenetetrazole induced changes in zebrafish behavior, neural activity and c-fos expression. Neuroscience 131, 759-768.

Bazaes, A., and Schmachtenberg, O. (2012). Odorant tuning of olfactory crypt cells from juvenile and adult rainbow trout. J. Exp. Biol. 215, 1740-1748.

Bepari, A. K., Watanabe, K., Yamaguchi, M., Tamamaki, N., and Hirohide, T. (2012). Visualization of odor-induced neuronal activity by immediate early gene expression. BMC Neurosci. 13:140. doi: 10.1186/1471-2202-13-140

Bianco, I. H., and Wilson, S. W. (2009). The habenular nuclei: a conserved asymmetric relay station in the vertebrate brain. Philos. Trans. R. Soc. Lond. B Biol. Sci. 364, 1005-1020.

Carboni, L., Carletti, R., Tacconi, S., Corti, C., and Ferraguti, F. (1998). Differential expression of SAPK isoforms in the rat brain. An in situ hybridisation study in the adult rat brain and during post-natal development. Mol. Brain Res. 60, 57-68.

Cirulli, F., Pistillo, L., De Acetis, L., Alleva, E., and Aloe, L. (1998). Increased number of mast cells in the central nervous system of adult male mice following chronic subordination stress. Brain Behav. Immun. 12, 123-133.

Close, R., Toro, S., Martial, J. A., and Muller, M. (2002). Expression of the zinc finger Egrl gene during zebrafish embryonic development. Mech. Dev. 118, 269-272.

Concha, M. L., Bianco, I. H., and Wilson, S. W. (2012). Encoding asymmetry within neural circuits. Nat. Rev. Neurosci. 13, 832-843.

Concha, M. L., Burdine, R. D., Russell, C., Schier, A. F., and Wilson, S. W. (2000). A nodal signaling pathway regulates the laterality of neuroanatomical asymmetries in the zebrafish forebrain. Neuron 28, 399-409.

Concha, M. L., Russell, C., Regan, J. C., Tawk, M., Sidi, S., Gilmour, D. T., et al. (2003). Local tissue interactions across the dorsal midline of the forebrain establish CNS laterality. Neuron 39, 423-438.

Concha, M. L., and Wilson, S. W. (2001). Asymmetry in the epithalamus of vertebrates. J. Anat. 199, 63-84.

Doldan, M. J., Cid, P., Mantilla, L., and De Miguel Villegas, E. (2011). Development of the olfactory system in turbot (Psetta maxima L.). J. Chem. Neuroanat. 41, 148-157.

Doving, K. B., Selset, R., and Thommesen, G. (1980). Olfactory sensitivity to bile acids in salmonid fishes. Acta Physiol. Scand. 108, 123-131.

Facchin, L., Burgess, H. A., Siddiqi, M., Granato, M., and Halpern, M. E. (2009). Determining the function of zebrafish epithalamic asymmetry. Philos. Trans. R. Soc. Lond. B Biol. Sci. 364, 1021-1032.

Frasnelli, E., Anfora, G., Trona, F., Tessarolo, F., and Vallortigara, G. (2010). Morpho-functional asymmetry of the olfactory receptors of the honeybee (Apis mellifera). Behav. Brain Res. 209, 221-225.

Friedrich, R. W., and Korsching, S. I. (1998). Chemotopic, combinatorial, and noncombinatorial odorant representations in the olfactory bulb revealed using a voltagesensitive axon tracer. J. Neurosci. 18 9977-9988.

Fuller, C. L., Yettaw, H. K., and Byrd, C. A. (2006). Mitral cells in the olfactory bulb of adult zebrafish (Danio rerio): morphology and distribution. J. Comp. Neurol. 499, 218-230.

Gamse, J. T., Kuan, Y. S., Macurak, M., Brosamle, C., Thisse, B., Thisse, C., et al. (2005). Directional asymmetry of the zebrafish epithalamus guides dorsoventral innervation of the midbrain target. Development 132, 4869-4881.

Gamse, J. T., Shen, Y. C., Thisse, C., Thisse, B., Raymond, P. A., Halpern, M. E., et al. (2002). Otx5 regulates genes that show circadian expression in the zebrafish pineal complex. Nat. Genet. 30, 117-121.

Gamse, J. T., Thisse, C., Thisse, B., and Halpern, M. E. (2003). The parapineal mediates left-right asymmetry in the zebrafish diencephalon. Development 130, 1059-1068.

Gaudry, Q., Hong, E. J., Kain, J., De Bivort, B. L., and Wilson, R. I. (2013). Asymmetric neurotransmitter release enables rapid odour lateralization in Drosophila. Nature 493 424-428.

Gilmour, D. T., Maischein, H. M., and Nusslein-Volhard, C. (2002). Migration and function of a glial subtype in the vertebrate peripheral nervous system. Neuron 34 577-588.

Gorelick, D. A., Watson, W., and Halpern, M. E. (2008). Androgen receptor gene expression in the developing and adult zebrafish brain. Dev. Dyn. 237, 2987-2995.

Guthrie, K. M., Anderson, A. J., Leon, M., and Gall, C. (1993). Odor-induced increases in c-fos mRNA expression reveal an anatomical "unit" for odor processing in olfactory bulb. Proc. Natl. Acad. Sci. U.S.A. 90, 3329-3333.

Guthrie, K. M., and Gall, C. M. (1995). Functional mapping of odoractivated neurons in the olfactory bulb. Chem. Senses 20, 271-282.

Hansen, A., and Finger, T. E. (2000). Phyletic distribution of crypt-type olfactory receptor neurons in fishes. Brain Behav. Evol. 55, 100-110.

Hansen, A., and Zeiske, E. (1998). The peripheral olfactory organ of the zebrafish, Danio rerio: an ultrastructural study. Chem. Senses 23, 39-48.

Hendricks, M., and Jesuthasan, S. (2007). Asymmetric innervation of the habenula in zebrafish. J. Comp. Neurol. 502, 611-619.
Herrick, J. C. (1948). The Brain of the Tiger Salamander, Ambystoma tigrinum. Chicago, IL: University of Chicago Press.

Hikosaka, O. (2010). The habenula: from stress evasion to valuebased decision-making. Nat. Rev. Neurosci. 11, 503-513.

Hikosaka, O., Sesack, S. R., Lecourtier, L., and Shepard, P. D. (2008). Habenula: crossroad between the basal ganglia and the limbic system. J. Neurosci. 28, 11825-11829.

Isogai, Y., Si, S., Pont-Lezica, L., Tan, T., Kapoor, V., Murthy, V. N., et al. (2011). Molecular organization of vomeronasal chemoreception. Nature 478, 241-245.

Kazi, J., Shiro, M., Kuchiiwa, S., and Nakagawa, S. (2004). Prolonged expression of $\mathrm{c}$-Fos protein in the lateral habenular nucleus of the japanese monkey (Macaca fuscata) after eye enucleation. Neurosignals 13, 130-133.

Kobayashi, Y., Sano, Y., Vannoni, E., Goto, H., Suzuki, H., Oba, A., et al. (2013). Genetic dissection of medial habenula-interpeduncular nucleus pathway function in mice. Front. Behav. Neurosci. 7:17. doi: 10.3389/ fnbeh.2013.00017

Kuan, Y. S., Gamse, J. T., Schreiber, A. M., and Halpern, M. E. (2007a). Selective asymmetry in a conserved forebrain to midbrain projection. J. Exp. Zool. 308B, 669-678.

Kuan, Y. S., Yu, H. H., Moens, C. B., and Halpern, M. E. (2007b). Neuropilin asymmetry mediates a left-right difference in habenular connectivity. Development 134, 857-865.

Lau, B. Y., Mathur, P., Gould, G. G., and Guo, S. (2011). Identification of a brain center whose activity discriminates a choice behavior in zebrafish. Proc. Natl. Acad. Sci. U.S.A. 108, 2581-2586.

Lauter, G., Soll, I., and Hauptmann, G. (2011). Multicolor fluorescent in situ hybridization to define abutting and overlapping gene expression in the embryonic zebrafish brain. Neural Dev. 6:10. doi: 10.1186/17498104-6-10

Lee, A., Mathuru, A. S., Teh, C., Kibat, C., Korzh, V., Penney, T. B., et al. (2010). The habenula prevents helpless behavior in larval zebrafish. Curr. Biol. 20, 2211-2216.

Letzkus, P., Ribi, W. A., Wood, J. T., Zhu, H., Zhang, S. W., and Srinivasan, M. V. (2006). Lateralization of olfaction in the honeybee Apis mellifera. Curr. Biol. 16, 1471-1476.

Liang, J. O., Etheridge, A., Hantsoo, L., Rubinstein, A. L., Nowak, S J., Belmonte, J. C. I., et al. (2000). 
Asymmetric nodal signaling in the zebrafish diencephalon positions the pineal organ. Development 127 , 5101-5112.

Mathuru, A. S., Kibat, C., Cheong, W. F., Shui, G., Wenk, M. R., Friedrich, R. W., et al. (2012). Chondroitin fragments are odorants that trigger fear behavior in fish. Curr. Biol. 22, 538-544.

Michel, W. C., and Lubomudrov, L. M. (1995). Specificity and sensitivity of the olfactory organ of the zebrafis, Danio rerio. J. Comp. Physiol. A 177, 191-199.

Michel, W. C., Sanderson, M. J., Olson, J. K., and Lipschitz, D. L. (2003). Evidence of a novel transduction pathway mediating detection of polyamines by the zebrafish olfactory system. J. Exp. Biol. 206, 1697-1706.

Miyasaka, N., Morimoto, K., Tsubokawa, T., Higashijima, S., Okamoto, H., and Yoshihara, Y. (2009). From the olfactory bulb to higher brain centers: genetic visualization of secondary olfactory pathways in zebrafish. J. Neurosci. 29, 4756-4767.

Muto, A., Ohkura, M., Kotani, T., Higashijima, S., Nakai, J., and Kawakami, K. (2011). Genetic visualization with an improved GCaMP calcium indicator reveals spatiotemporal activation of the spinal motor neurons in zebrafish. Proc. Natl. Acad. Sci. U.S.A. 108, 5425-5430.

Oka, Y., Saraiva, L. R., and Korsching, S. I. (2012). Crypt neurons express a single V1R-related ora gene. Chem. Senses 37, 219-227.

Okamoto, H., Agetsuma, M., and Aizawa, H. (2012). Genetic dissection of the zebrafish habenula, a possible switching board for selection of behavioral strategy to cope with fear and anxiety. Dev. Neurobiol. 72, 386-394.

Okuyama, T., Suehiro, Y., Imada, H., Shimada, A., Naruse, K., Takeda, H., et al. (2011). Induction of c-fos transcription in the medaka brain (Oryzias latipes) in response to mating stimuli. Biochem. Biophys. Res. Commun. 404, 453-457.

Paul, M. J., Indic, P., and Schwartz, W. J. (2011). A role for the habenula in the regulation of locomotor activity cycles. Eur. J. Neurosci. 34, 478-488.

Pfeiffer, W. (1977). The distribution of fright reaction and alarm substance cells in fishes. Copeia 4, 653-665.
Polkinghorne, C. N., Olson, J. M., Gallaher, D. G., and Sorensen, P. W. (2001). Larval sea lamprey release two unique bile acids to the water at a rate sufficient to produce detectable riverine pheromone plumes. Fish Physiol. Biochem. 24, 15-30.

Prasada Rao, P. D., and Finger, T. E. (1984). Asymmetry of the olfactory system in the brain of the winter flounder, Pseudopleuronectes americanus. J. Comp. Neurol. 225, 492-510.

Ramón y Cajal, S. (1995). Histology of the Nervous System of Man and Vertebrates. Translated from the French by N. Swanson and L. W. Swanson. New York, NY: Oxford University Press.

Regan, J. C., Concha, M. L., Roussigne, M., Russell, C., and Wilson, S. W. (2009). An Fgf8-dependent bistable cell migratory event establishes CNS asymmetry. Neuron 61, 27-34.

Rogers, L. J., and Vallortigara, G. (2008). From antenna to antenna: lateral shift of olfactory memory recall by honeybees. PLOS ONE 3:e2340. doi: 10.1371/journal.pone.0002340

Rolen, S. H., Sorensen, P. W., Mattson, D., and Caprio, J. (2003). Polyamines as olfactory stimuli in the goldfish Carassius auratus. J. Exp. Biol. 206, 1683-1696.

Sallaz, M., and Jourdan, F. (1993). C-fos expression and 2-deoxyglucose uptake in the olfactory bulb of odour-stimulated awake rats. Neuroreport 4, 55-58.

Schmachtenberg, O. (2006). Histological and electrophysiological properties of crypt cells from the olfactory epithelium of the marine teleost Trachurus symmetricus. J. Comp. Neurol. 495, 113-121.

Signore, I. A., Guerrero, N., Loosli, F., Colombo, A., Villalon, A., Wittbrodt, J., et al. (2009). Zebrafish and medaka: model organisms for a comparative developmental approach of brain asymmetry. Philos. Trans. R. Soc. Lond. B Biol. Sci. 364, 991-1003.

Silver, R., Silverman, A. J., Vitkovic, L., and Lederhendler, I. I. (1996). Mast cells in the brain: evidence and functional significance. Trends Neurosci. 19, 25-31.

Snelson, C. D., Santhakumar, K., Halpern, M. E., and Gamse, J. T. (2008). Tbx2b is required for the development of the parapineal organ. Development 135, 1693-1702.

Speedie, N., and Gerlai, R. (2008) Alarm substance induced behavioral responses in zebrafish (Danio rerio). Behav. Brain Res. 188 , 168-177.

Sugama, S., Cho, B. P., Baker, H., Joh, T. H., Lucero, J., and Conti, B. (2002). Neurons of the superior nucleus of the medial habenula and ependymal cells express IL-18 in rat CNS. Brain Res. 958, 1-9.

Sutherland, R. J. (1982). The dorsal diencephalic conduction system - a review of the anatomy and functions of the habenular complex. Neurosci. Biobehav. Rev. 6, 1-13.

Tabor, R., and Friedrich, R. W. (2008). Pharmacological analysis of ionotropic glutamate receptor function in neuronal circuits of the zebrafish olfactory bulb. PLOS ONE 3:e1416. doi 10.1371/journal.pone.0001416

Taylor, R. W., Qi, J. Y., Talaga, A. K., Ma, T. P., Pan, L., Bartholomew, C. R., et al. (2011). Asymmetric inhibition of Ulk2 causes left-right differences in habenular neuropil formation. J. Neurosci. 31, 9869-9878.

Thisse, B., Thisse, C. (2004). Fast Release Clones: A High Throughput Expression Analysis. ZFIN Direct Data Submission (http://zfin.org). Available online at: http://zfin. org/cgi-bin/webdriver?MIval=aapubview2.apg\&OID=ZDB-PUB040907-1

Velez, Z., Hubbard, C. A., Barata, E. N., and Canario, A. V. M. (2005). Evidence for functional asymmetry in the olfactory system of the senegalese sole (Solea senegalensis). Physiol. Biochem. Zool. 78, 756-765.

Velez, Z., Hubbard, P. C., Barata, E. N., and Canario, A. V. M. (2007). Differential detection of conspecific-derived odorants by the two olfactory epithelia of the Senegalese sole (Solea senegalensis). Gen. Comp. Endocrinol. 153, 418-425.

Vielma, A., Ardiles, A., Delgado, L., and Schmachtenberg, O. (2008). The elusive crypt olfactory receptor neuron: evidence for its stimulation by amino acids and CAMP pathway agonists. J. Exp. Biol. 211, 2417-2422.

Vitebsky, A., Reyes, R., Sanderson, M. J., Michel, W. C., and Whitlock, K. E. (2005). Isolation and characterization of the laure olfactory behavioral mutant in the zebrafish, Danio rerio. Dev. Dyn. 234, 229-242.

Vize, P. D., Mccoy, K. E., and Zhou, X. (2009). Multichannel wholemount fluorescent and fluorescent/chromogenic in situ hybridization in Xenopus embryos. Nat. Protoc. 4, 975-983.

Waldman, B. (1982). Quantitative and developmental analyses of the alarm reaction in the Zebra Danio, Brachydanio rerio. Copeia 1, 1-9.

Walker, C. (1999). Haploid screens and gamma-ray mutagenesis. Methods Cell Biol. 60, 43-70.

Wes, P. D., and Bargmann, C. I. (2001). C. elegans odour discrimination requires asymmetric diversity in olfactory neurons. Nature 410, 698-701.

Westerfield, M. (2000). The Zebrafish Book. Eugene, OR: University of Oregon Press.

Wilhelm, M. (2011). Neuro-immune interactions in the dove brain. Gen. Comp. Endocrinol. 172, 173-180.

Zhang, F. Q., Zhou, W. H., Liu, H. F., Zhu, H. Q., Tang, S., Lai, M. J., et al. (2005). Increased c-Fos expression in the medial part of the lateral habenula during cue-evoked heroin-seeking in rats. Neurosci. Lett. 386, 133-137.

Conflict of Interest Statement: The authors declare that the research was conducted in the absence of any commercial or financial relationships that could be construed as a potential conflict of interest.

Received: 16 February 2013; paper pending published: 06 March 2013; accepted: 02 May 2013; published online: 21 May 2013.

Citation: deCarvalho TN, Akitake CM, Thisse C, Thisse $B$ and Halpern $M E$ (2013) Aversive cues fail to activate fos expression in the asymmetric olfactoryhabenula pathway of zebrafish. Front. Neural Circuits 7:98. doi: 10.3389/fncir. 2013.00098

Copyright (C) 2013 deCarvalho, Akitake, Thisse, Thisse and Halpern. This is an open-access article distributed under the terms of the Creative Commons Attribution License, which permits use, distribution and reproduction in other forums, provided the original authors and source are credited and subject to any copyright notices concerning any thirdparty graphics etc. 